\title{
Selection of Anti-gluten DNA Aptamers in a Deep Eutectic Solvent
}

\author{
Rossella Svigelj, ${ }^{[b]}$ Nicolò Dossi, ${ }^{[b]}$ Rosanna Toniolo, ${ }^{[b]}$ Rebeca Miranda-Castro, ${ }^{[a]}$ Noemí de-los- \\ Santos-Álvarez, ${ }^{[a]}$ and M. Jesús Lobo-Castañón*[a]
}

\begin{abstract}
Here we show the feasibility of using deep eutectic solvents as a faster way of selecting aptamers targeting poorly water-soluble species. This unexplored concept is illustrated for gluten proteins. In this way, detection of gluten based on aptamers can be performed directly in the extraction media with improved detectability. We envision deep implications not only for applications in food safety control but also in biomedicine.
\end{abstract}

Systematic evolution of ligands by exponential enrichment (SELEX) has proven to be a powerful technology for developing the nucleic acid synthetic binders called aptamers. ${ }^{[1]}$ SELEX mimics natural selection and it is based on a series of iterative selection and amplification steps, starting from a chemically synthesized pool of different nucleic acid sequences that are challenged to bind to a desired target under defined conditions. Despite the conditions used in the interaction step of the SELEX are the optimal for operation in the aptamer-target interaction, until now most of the selection processes have been carried out in aqueous media, with only a few exceptions challenging the aptamer interaction in mixtures of aqueous buffer and organic solvents (up to $25 \%$ ). ${ }^{[2]}$ In consequence, aptamers are of limited utility for the recognition of poorly water soluble species. Motivated by this observation, we demonstrate here that SELEX can be performed in high solubilizing capacity solvents named deep eutectic solvents (DESs).

DESs are a class of ionic liquids formed by eutectic mixtures of a quaternary ammonium salt and a hydrogen bond donor such as choline chloride and 1,2-ethanediol. Since they were firstly reported by Abbott et al., ${ }^{[3]}$ they proved to be excellent solvents for a wide range of molecules with medium to low polarity; they have been applied in many different fields such as organic synthesis, electrochemistry and biocatalysis. ${ }^{[4]}$ This new family of designer solvents can be easily tailored by choosing the nature and ratio of the hydrogen bond donor and acceptor components, while conserving the DNA structural integrity. ${ }^{[5]}$ Despite DESs allow the creation of functional DNA structures of greater complexity and different from those obtained in water, ${ }^{[6]}$ suggesting they may favor the folding of aptamers into functional shapes, no one has reported the aptamer selection in DESs yet.

[a] Dr. R. Miranda-Castro, Dr. N. de-los-Santos-Álvarez, Prof. M. J. Lobo-Castañón

Departamento de Química Física y Analítica

Universidad de Oviedo

Julián Clavería 8. 33006 Oviedo, Spain

E-mail: mjlc@uniovi.es

[b] MSc. R. Svigelj, Dr. N. Dossi, Prof. R. Toniolo

Department of Agrifood, Environmental and Animal Sciences University of Udine

via Cotonificio 108, 33100 Udine, Italy

Supporting information for this article is given via a link at the end of the document.
As a proof of principle, we have selected aptamers targeting gluten proteins, which are found in any food containing wheat, rye, barley and some oat varieties and are responsible for triggering celiac disease. Gluten actually describes a complex mixture of water-insoluble storage proteins, which are divided into two main fractions: prolamins and glutelins. Celiac disease-toxic and celiac disease-immunogenic peptides have been identified from all these proteins, ${ }^{[7]}$ so to ensure the safety of foodstuffs for celiac patients analytical methods should be able to detect all of them. However, the development of such methods has been hampered by the limited efficiency and reproducibility of gluten extraction protocols. ${ }^{[8]}$ Recently, it has been shown that the DES named ethaline, a mixture of choline chloride and 1,2-ethanediol (1:2), is a very effective medium for gluten solubilization. ${ }^{[9]}$ However, as it is the case with the classical extraction media, large dilutions are required to make them compatible with the antibodies commonly used as specific receptors. As such, the development of aptamers able to recognize the extracted proteins directly in DES media without degrading sensitivity could improve their utility.

For aptamer selection the proteolytically resistant 33-mer peptide, which is a highly hydrophobic peptide identified as one of the most immunogenic fragments from $\alpha 2$-gliadin, ${ }^{[10]}$ with a biotin tag in its C-terminus, was immobilized onto streptavidin modified magnetic particles (MPs) to perform a magnetic beadbased selection protocol. ${ }^{[11]}$ The colloidal stability of the modified-MP suspension in both aqueous buffer and ethaline was monitored by multiple light scattering (Supporting Information, Figure S1). While relatively fast aggregation of MPs was observed in the aqueous buffer, with a sedimentation speed of $0.06 \mathrm{~mm} / \mathrm{h}$ in $24 \mathrm{~h}$, the DES led to a homogeneous distribution of the modified microparticles through the entire measurement cell for as long as $24 \mathrm{~h}$. This stabilization reflects the high solubilizing ability of DES and greatly facilitates the MPs manipulation.

The first SELEX round was performed by incubating 1 nmol of a starting library of 80-mer DNA oligonucleotides with a central 40-mer random region flanked by primer binding sequences (Table S1) with $20 \mu \mathrm{g}$ of the microparticles with the immobilized peptide in ethaline ( $1 \mathrm{~mL}$ total volume); $0.1 \mathrm{nmol}$ of tRNA were incorporated as a competitor, and the unspecific binding of oligonucleotides was minimized using BSA in the ethaline solution. After interaction for $60 \mathrm{~min}$ the bound oligonucleotides fraction was magnetically separated and eluted with hot water. The recovered oligonucleotides were then amplified by PCR with a biotinylated reverse primer; the amplification products were entrapped onto streptavidin-modified MPs, and denatured with $\mathrm{NaOH}$ to prepare a new pool for further rounds of selection. All the process was repeated under progressively increased stringency (Table S2). Sequences with affinity for the support were eliminated with a counter selection before round 3 , by incubating the ssDNA pool from the previous round with streptavidin-modified MPs. 

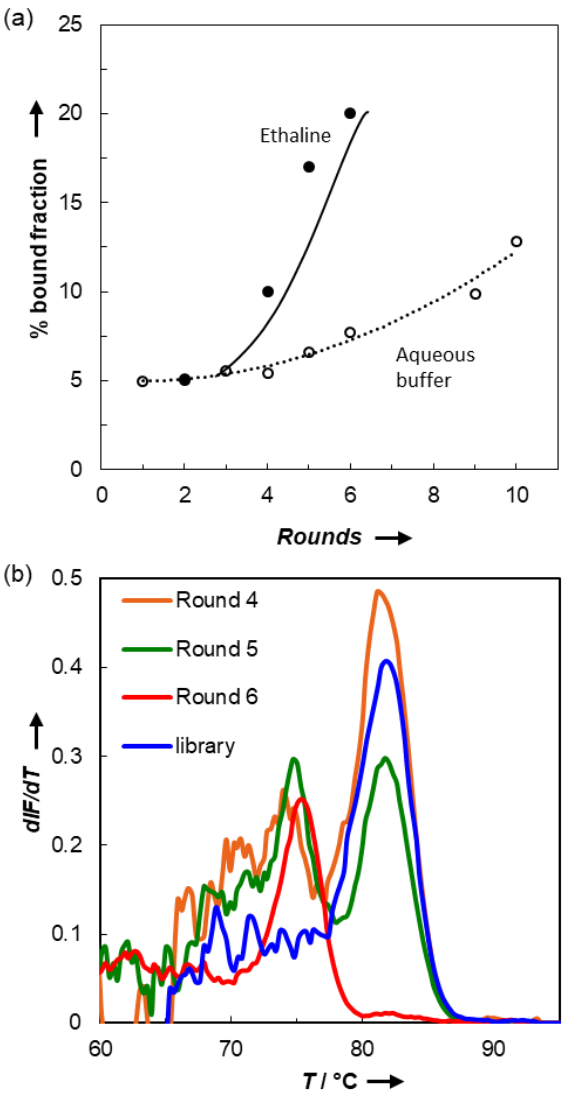

Figure 1. (a) Evolution of the percentage of oligonucleotides bound to the 33 mer-modified MPs throughout the SELEX rounds depending on whether the selection was performed in ethaline or aqueous buffer. The percentage of enrichment was obtained by absorbance measurements at $260 \mathrm{~nm}$ when SELEX was performed in ethaline, and fluorescence measurements for the evolution in aqueous buffer. (b) Melting curves after total denaturation at $95{ }^{\circ} \mathrm{C}$ for $30 \mathrm{~s}$, and $30 \mathrm{~s}$ reannealing at $50^{\circ} \mathrm{C}$ of the initial library and pools after the specified selection rounds in ethaline.

To determine how the ethaline medium affects the efficiency of the selection, we compared the rate of enrichment of the successive ssDNA pools in sequences with affinity towards the immunotoxic peptide with the SELEX evolution using similar selection conditions but in aqueous buffer..$^{[12]}$ Since the first rounds of selection in ethaline, we observed an enrichment of the initial library significantly faster than that obtained in aqueous medium, with a roughly 3 -fold increase with respect to the aqueous selection after six rounds (Figure 1a). This presumably occurs because of the high viscosity of the DES, which imposed more stringent conditions during selection, and the potential ability of DES to alter the secondary structure of nucleic acids ${ }^{[5]}$ given an additional structural selection.

The enrichment after six rounds of selection in ethaline was greater than after 10 rounds in water, and it was already leveling-off. Agarose gel electrophoresis analysis revealed that two different set of products appeared after the fourth cycle: the expected 80-mer and shorter products. Despite the additional rounds of selection were performed with only the oligonucleotides of the expected length, purified by gel electrophoresis, the shorter products continued to increase, and they were the only appearing after the sixth round of selection. To confirm the decrease in diversity during SELEX the remelting profiles $^{[13]}$ of the successive DNA pools were analysed (Figure 1b). A biphasic distribution of sequences is observed after round four, but unexpectedly the growing remelting peak appeared at a temperature $\left(T_{m}=75{ }^{\circ} \mathrm{C}\right)$ lower than the remelting peak in the original library $\left(\mathrm{T}_{\mathrm{m}}=81^{\circ} \mathrm{C}\right)$. This is not compatible with the usual PCR artefacts arising from cross-hybridization of amplified library members, which once extended by polymerase give byproducts longer than expected and detrimental to the enrichment process. ${ }^{[14]}$ The presence of shortened sequences, with lower melting temperature, may stem from template shifting during amplification ${ }^{[15]}$ pushed by the stabilization of certain secondary structures in DES. They were not observed when the same primers were employed in the selection of anti-33-mer aptamers in aqueous buffer. In addition, PCR amplification of the starting DNA library gave products of the expected size even in the presence of $1 \%$ of ethaline, thus confirming that the polymerase is active in the presence of DES. Although the appearance of short sequences was not detrimental for the enrichment, we decided to analyse ssDNA surviving after the fifth cycle by highthroughput sequencing using the Illumina platform.

The raw reads were filtered on the bases of the quality of their constant regions. We obtained more than 830000 sequences; most of them are $40 \mathrm{nt}$ in length (after removing their constant regions), with only $0.3 \%$ of sequences with lengths between 18 and $22 \mathrm{nt}$ (centered at $20 \mathrm{nt}$ ). The most probable origin of these short sequences is failure in PCR amplification as they have a high GC content. The 40-mer oligonucleotides were analysed by frequency and consensus structural motifs with the Galaxy web platform. ${ }^{[16]}$ Interestingly, we found that the most abundant sequence, Gli1, is the same one we identified as the most represented in a SELEX process against the 33-mer peptide in aqueous buffer. ${ }^{[12]}$ The highest affinity aptamer in aqueous media, Gli4, is also among the more represented members in the SELEX in ethaline. Additionally, by analysing the structural features of the potential aptamers, we identified two consensus sequences residing in hairpin structures. The first one is an $8 \mathrm{nt}$ motif (TGTCTGAA, Table S3) that appears in 518 different sequences, including Gli1 and Gli4, with the core $6 \mathrm{nt}$ motif GTCTGA, in the loop of the hairpin structures, repeated in $1 \%$ of the filtered sequences. The second one is a 9 nt motif (AGCTACAAC, Table S3), which is common to 61 different sequences, including Gli1. The sequences Gli2D and Gli3D (Table S3) were selected as additional representatives of each group because they are characterized by the minimum free energy secondary structure in their respective group (Figure S2 and Supplementary Information). The four sequences included in Table S3 were the candidate aptamers selected for further functional characterization in ethaline solutions.

The affinity of the selected aptamers for both the 33-mer peptide and the whole gliadin was estimated using a MP-based binding assay with chronoamperometric detection. Due to the lack of an accepted reference standard for gluten, PWGgliadin, ${ }^{[17]}$ which is the most commonly used for gluten calibration in commercial kits, was covalently linked to tosylactivated MPs. We first titrated both 33-mer modified MPs as 
those employed during the SELEX process, and gliadin-modified MPs, with increasing amounts of the aptamers, with a biotin tag at the 5'-terminus, in ethaline. To measure the bound aptamers, we then used the streptavidin-peroxidase (POD) conjugate, and after entrapping the MPs onto screen-printed carbon electrodes, the POD activity was monitored at $0 \mathrm{~V}$ (Supporting Information). The observed current increases with increasing aptamer concentration when all the aptamers were challenged to the immobilized 33-mer and the native protein (Figure 2). The resulting binding curves were fitted to the Hill equation, giving the dissociation constants summarized in Table 1. Gli1 and Gli4 retained their activity to recognize both the peptide and the whole protein in ethaline. In contrast to aqueous buffer, where Gli4 exhibited the highest affinity and the same behavior for both targets, ${ }^{[12]}$ in ethaline only Gli1 was able to bind with the same affinity to PWG and the 33-mer peptide. The use of DESs as selection media, where both the aptamer and target may adopt conformations different from those shown in water, could therefore be an advantage.

Table 1. Dissociation constants $(\mu \mathrm{M})$ of complexes of the aptamers with 33mer peptide and protein.

\begin{tabular}{lllll}
\hline & Gli1 & Gli4 & Gli3D & Gli2D \\
\hline 33-mer & $1.4 \pm 0.1$ & n.d. [a] & $1.3 \pm 0.1$ & n.d. [a] \\
PWG & $1.4 \pm 0.2$ & $1.3 \pm 0.4$ & 25 & 13 \\
\hline
\end{tabular}

[a] Not determined owing to a poor fitting to the Hill equation

The most abundant sequence, Gli1, is also the best binder in ethaline regardless the peptide used as target is included within the natural protein. This is also in contrast to the previous aqueous SELEX, presumably due to the kinetic constrains imposed by the DES. Surprisingly, Gli4, which was the best binder in aqueous buffer, displayed in ethaline similar affinity to Gli1 towards PWG, although with $\sim 75 \%$ of signal at saturation, but relatively poor affinity to 33-mer peptide. Saturation level was not obtained for the 33-mer peptide-Gli4 system at concentrations of aptamer in the range of our assay (up to $5 \mu \mathrm{M}$ ), and consequently we were unable to estimate the affinity constant. These results may be indicative of a change in the secondary structure of DNA in ethaline, in agreement with our hypothesis that DES may favor a structural selection of folded states that may be not accessed in aqueous solutions. The aptamer Gli3D displayed the opposite behavior, with the same affinity as the best binder towards the peptide but $\sim 10$ fold reduced affinity to the whole protein. It is significant to note that Gli4 and Gli3D belong to different groups regarding the two most abundant identified motifs, whereas Gli1 contains both motifs. The other aptamer, Gli2D, including only the first motif, displayed poor affinity towards both the peptide and the protein. This means that the presence of one of the motifs allows the recognition of the peptide within the native protein but the affinity is one order of magnitude superior when both are present. Note that a variation of the second motif also appears in Gli4. Recognition of 33-mer with high affinity requires the second motif. Those findings could guide the refinement of aptamers by truncation
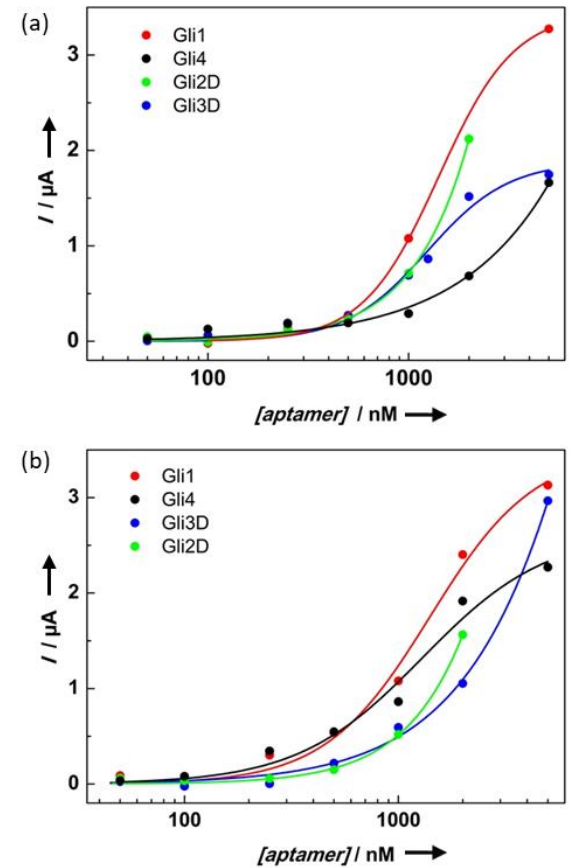

Figure 2. Binding curves of biotinylated selected aptamers to (a) biotinylated 33-mer linked to streptavidin-modified magnetic particles, and (b) PWG covalently bound to tosyl-activated magnetic particles. The data are obtained from chronoamperometric measurements at $0 \mathrm{~V}$ of the aptamer-bound fraction after labeling with streptavidin-POD, using tetramethylbenzidine and $\mathrm{H}_{2} \mathrm{O}_{2}$ as substrates.

To further confirm the ability of DES to stabilize different DNA secondary structures, we characterized Gli4 and Gli1 aptamer solutions in aqueous buffer and ethaline using circular dichroism (CD) spectroscopy (Figure S3). Samples on aqueous buffer corresponded to DNA conformations whose CD spectra only show maxima around $275 \mathrm{~nm}$ and minima around $245 \mathrm{~nm}$, which suggests B-helix hairpins, in agreement to the secondary structure of the aptamers predicted by using Mfold, ${ }^{[18]}$ although only Gli4 displayed a conservative spectrum. The CD of the oligonucleotides in ethaline (Figure S3b) changes dramatically, with a characteristic negative band at short wavelengths ( 220 $225 \mathrm{~nm}$ ) consistent with a change to a conformation of triple helices. ${ }^{[19]}$ The negative peak, indicative of a Hoogsteen basepair interaction, is accompanied by a band around $280 \mathrm{~nm}$ characterizing the presence of the Watson-Crick intramolecular duplex. The triplex formation, which is a water releasing reaction, may be favored by the dehydration conditions imposed by DES. ${ }^{[5]}$ Additional features of CD spectra in ethaline are possibly due to the presence of additional structures.

A competitive assay confirmed the usefulness of the selected aptamers for the direct detection of gluten in ethaline extracts, without extra-dilutions. We used 33-mer-modified magnetic particles and a limiting amount of biotinylated-Gli1 aptamer 
(Figure 3 and Supporting Information). The presence of gliadin in the ethaline solution effectively reduces the aptamer binding to $64 \%$ for a PWG concentration as low as $0.1 \mathrm{ppm}$, with a further decrease in binding to $50 \%$ as PWG increases to $1 \mathrm{ppm}$, suggesting that the signal change arises due to the specific displacement of the bound aptamer by PWG in solution. Consistent with this, the binding remained unchanged when the assay is performed with ethaline extracts of rice flour.

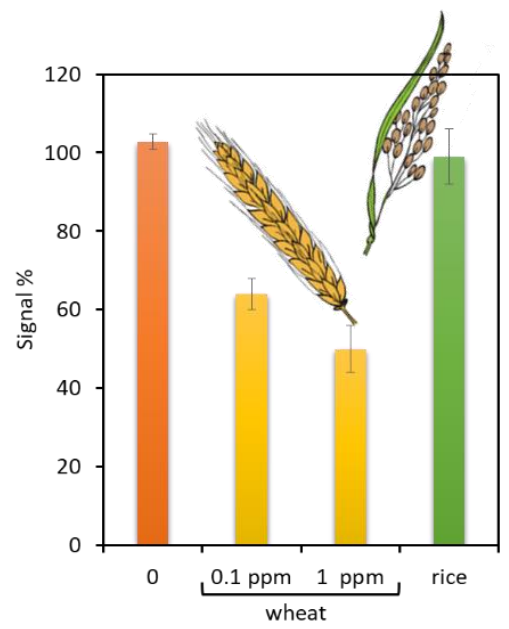

Figure 3. Signal change in a competitive assay, using $1 \mu \mathrm{M}$ Biotin-Gli1 and $0.1 \mathrm{mg} / \mathrm{mL}$ of $33-\mathrm{mer}$ modified magnetic beads, for different amounts of standard gliadin in DES and ethaline extracts of rice flour.

The development of aptamers against gluten/gliadin is a longawaited goal where many researchers have failed as it has been openly recognized. ${ }^{[20]}$ Herein, we take a step further by demonstrating the viability of a SELEX in a green extraction solvent for the first time, thus providing a method for obtaining aptamers able to recognize non-soluble and poorly watersoluble molecules or species prone to aggregation in aqueous solutions. This have deep implications in the way the gluten analysis is currently performed, e.g. avoiding dilution so pushing down the limit of detection and outperforming the extraction yield The selection in DESs has proved to be faster than in aqueous media, reducing the number of selection cycles needed for achieving a similar enrichment factor from the starting DNA library. The high viscosity of DESs may impose additional restrictions during the selection, which added to the trapping of some folded states stabilized through a diffusion limited process, ${ }^{[6 a]}$ propel the selection. Owing to its high efficiency while mimicking the intracellular media, the application of DESSELEX to the selection of aptamers against a wide variety of targets of interest for biomedical applications, including intracellular targets, is enticing.

\section{Acknowledgements}

This research was supported by the Spanish Ministerio de Economía y Competitividad through the Project CTQ201563567-R, Principado de Asturias government (Project FC15GRUPIN-14-025) and co-financed by FEDER funds. Financial support from the Italian Ministry of University and Scientific research is also gratefully acknowledged. We thank Dr. Alessandra Corazza, of the Department of Medicine at the University of Udine, for assistance with Circular Dichroism measurements.

Keywords: aptamers $•$ ionic liquids $•$ gluten $\cdot S E L E X \cdot$ waterinsoluble targets

[1] a) M. Darmostuk, S. Rimpelova, H. Gbelcova, T. Ruml, Biotechnol. Adv. 2015, 33, 1141-1161; b) M. R. Dunn, R. M. Jimenez, J. C. Chaupt, Nat Rev. Chem. 2017, 1, 0076 .

[2] a) T. Chaou, B. Vialet, L. Azéma, Methods 2016, 97, 11-19; b) I. Machado, V. C. Özalp, T. Schäfer, Chem. Eur. J. 2014, 20, 11820 11825.

[3] A. P. Abbott, D. Boothby, G. Capper, D. L. Davies, R. K.; Rasheed, J Am. Chem. Soc. 2004, 126, 9142-9147.

[4] a) M. Francisco, A. van den Bruinhorst, M.C. Kroon, Angew. Chem 2013, 125, 3152-3163; Angew. Chem. Int. Ed. 2013, 52, 3074-3085; b) Y. Dai, J. van Sponsen, G.- J. Witkamp, R. Verpoorte, Y. H. Choi, Anal. Chim. Acta 2013, 766, 61-68.

[5] I. Mamajanov, A. E. Engelhart, H. D. Bean, N. V. Hud, Angew. Chem 2010, 122, 6454-6458; Angew. Chem. Int. Ed. 2010, 49, 6310-6314.

[6] a) F. M. Lannan, I. Mamajanov, N. V. Hud, J. Am. Chem. Soc. 2012 134, 15324-15330; b) A. Gállego, M. A. Grover, N. V. Hud, Angew. Chem. 2015, 127, 6869-6873; Angew. Chem. Int. Ed. 2015, 54, 67656769.

[7] J. A. Tye-Din, J. A.Stewart, J. A. Dromey, T. Beissbarth, D. A. van Heel, A. Tatham, K. Henderson, S. I. Mannering, C. Gianfrani, D. P. Jewell, A. V. S. Hill, J. McCluskey, J. Rossjohn, R. P. Anderson, Sci. Transl. Med. 2010, 2, 41ra51.

[8] A. Fallahbaghery, W. Zou, K. Byrne, C. A. Howitt, M. L., Colgrave, J. Agric. Food Chem. 2017, 65, 2857-2866.

[9] a) R. Svigelj, R. Bortolomeazzi, N. Dossi, A. Giacomino, G. Bontempelli, R. Toniolo, Food Anal. Methods 2017, 10, 4079-4085; b) F. Lores, V Romero, I. Costas, C. Bendicho, I. Lavilla, Talanta 2017, 162, 453-459.

[10] L. Shan, Ø. Molberg, I. Parrot, F. Hausch, F. Filiz, G. M. Gray, L. M. Sollid, C. Khosla, Science 2002, 297, 2275-2279.

[11] R. Stoltenburg, C. Reineman, B. Strehlitz, Anal. Bioanal. Chem. 2005 383, 83-91.

[12] a) S. Amaya-González, N. de-los-Santos-Álvarez, A. J. MirandaOrdieres, M. J. Lobo-Castañón, Anal. Chem. 2014, 86, 2733-2739; b) N. de-los Santos Álvarez, S. Amaya-González, A.J. Miranda-Ordieres, M. J. Lobo-Castañón patent ES2436861 B2 (May 31, 2012).

[13] J. Vanbrabant, K. Leirs, K. Vanschoenbeek, J. Lammertyn, L. Michiels, Analyst 2014, 139, 589-595.

[14] F. Tolle, J. Wilke, J. Wengel, G. Mayer, PLoS One 2014, 9, e114693.

[15] M. U. Musheev, S. N. Krylov, Anal. Chim. Acta 2006, 564, 91-96.

[16] E. Afgan, D. Baker, M. van den Beek, D. Blankenberg, D. Bouvier, M. Cech, J. Chilton, D. Clements, N. Coraor, C. Eberhard, B. Grüning, A. Guerler, J. Hillman-Jackson, G. Von Kuster, E. Rasche, N. Soranzo, N. Turaga, J. Taylor, A. Nekrutenko, J. Goecks, Nucleic Acids Res. 2016, 44, W3-W10.

[17] R. van Eckert, E. Berghofer, P. J. Ciclitira, F. Chirdo, S. Denery-Papini, H. J. Ellis, P. Ferranti, P. Goodwin, U. Immer, G. Mamone, E. Mendez, T. Mothes, S. Novalin, A. Osman, M. Rumbo, M. Stern, L. Thorell, A. Whim, H. Wieser, J. Cereal Sci. 2006, 43, 331-341.

[18] M. Zuker, Nucleic Acids Res. 2003, 31, 3406-3415.

[19] a) A. M. Soto, J. Loo, L. A. Marky, J. Am. Chem. Soc. 2002, 124, 14355-14363; b) N. Sugimoto, P. Wu, H. Hara, Y. Kawamoto, Biochemistry 2001, 40, 9396-9405.

[20] A. Pinto, P. Nadal Polo, O. Henry, M.C. Bermudo Redondo, M. Svobodova, C.K. O’Sullivan, Anal. Bioanal. Chem. 2014, 406, 515-524. 


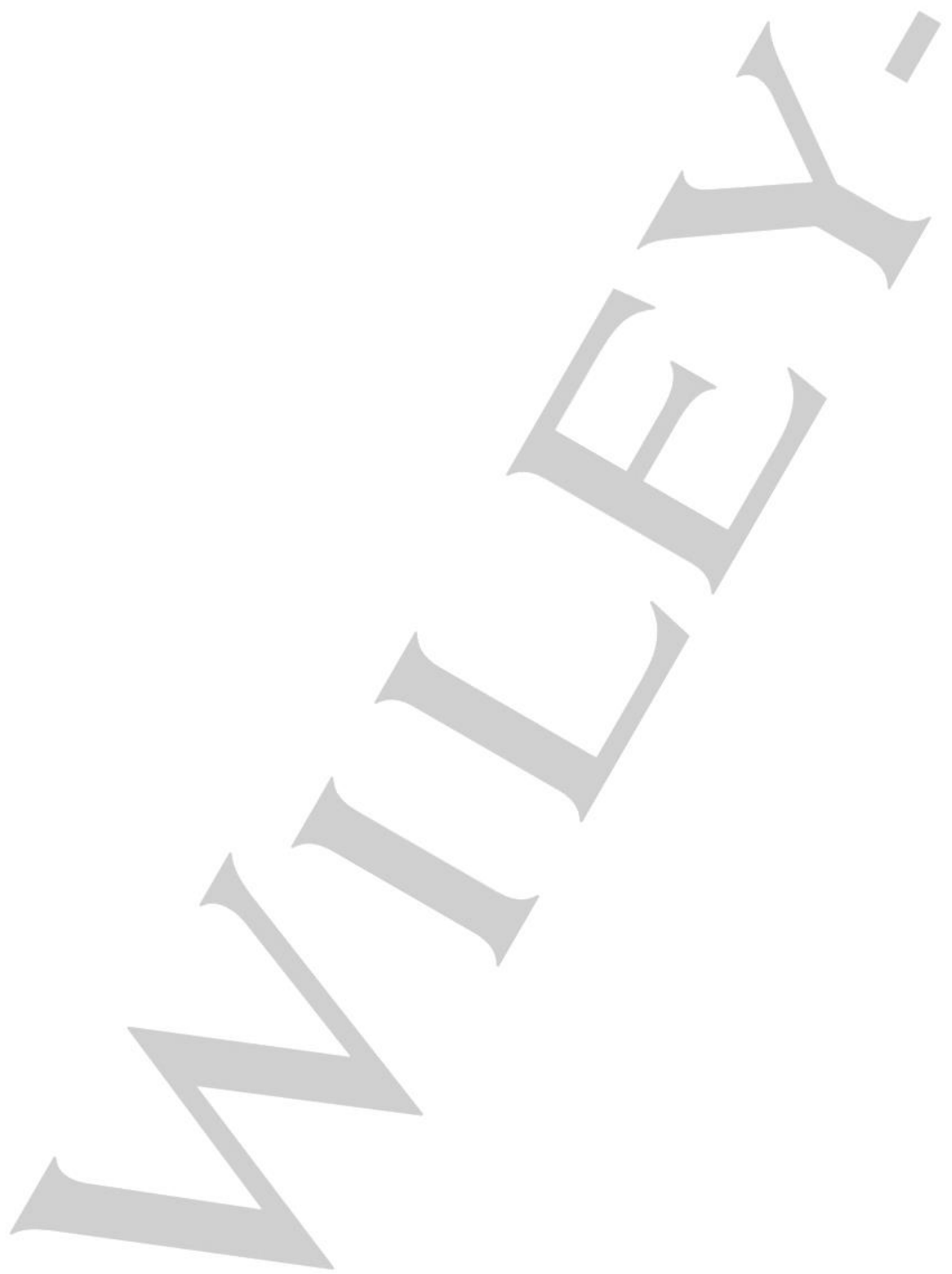




\section{COMMUNICATION}

DES-SELEX: aptamer selection and interaction without water. Aptamers can be selected in deep eutectic solvents composed of choline chloride and 1,2- ethanediol faster than in aqueous media. These highly solubilizing media, mimicking the intracellular environment, allow targeting non-soluble or poorly watersoluble species, which include targets of great interest in biomedical, pharmaceutical and food analysis.

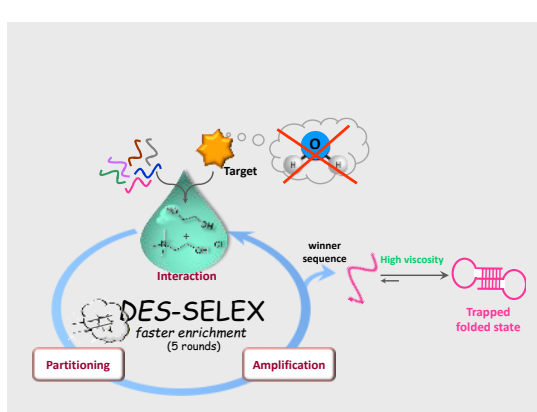

R. Svigelj, N. Dossi, R. Toniolo, $R$. Miranda-Castro, N. de-los-SantosÁlvarez, M. J. Lobo-Castañón*

Page No. - Page No.

Selection of anti-gluten aptamers in a deep-eutectic solvent 